\title{
Numerical Study of Entropy Wave Evolution within a HPT Stage
}

\author{
Lorenzo Pinelli ${ }^{1 *}$, Leonardo Lilli ${ }^{1}$, Andrea Arnone ${ }^{1}$, Paolo Gaetani $^{2}$ and Giacomo Persico ${ }^{2}$ \\ ${ }^{1}$ Department of Industrial Engineering, Università degli Studi di Firenze, Via di S. Marta 3, 50139 \\ Firenze, Italy \\ ${ }^{2}$ Energy Department, Politecnico di Milano, Via Lambruschini 4, 20158 Milano, Italy.
}

\begin{abstract}
Component reciprocal interaction and aero-thermal coupling are critical aspects in modern turbomachinery design. Combustors and highpressure turbine (HPT) interaction is extremely critical due to the compact and lightweight system design. In this context, computational and experimental analyses are thus necessary to study the interaction of the high temperature gas coming from combustor systems and entering the turbine in order to avoid engine mis-operations and to lower the indirect core noise generation. This paper presents a numerical study of pulsating temperature distortion (entropy wave) evolution within a high pressure turbine stage. Four different clocking positions between the 11 temperature spots and the 22 stators have been studied. The numerical results, obtained by URANS computations (TRAF code) and by a dedicated post-processing based on Fourier coefficients, have been compared with experimental measurements coming from the Laboratorio di Fluidodinamica delle Macchine (LFM) of the Politecnico di Milano (Italy) where the HP stage rig is located. The excellent agreement between numerical results and experimental acquisitions confirms the accuracy of the numerical approach. Such results also suggest recommendations for the thermal design of the rows and are the main prerequisite for the study of the indirect core noise generation.
\end{abstract}

\section{Introduction}

The restrictions imposed by international regulation authority in terms of pollutant emissions have pushed the turbomachinery combustor design towards a lean-premixed combustion process and compact layouts with short axial gaps between the combustor and the first turbine nozzle [1]. As a direct consequence, the interaction of these two components in terms of velocity, pressure and temperature fluctuations of the flow coming from combustor exhaust and entering the turbine, has become a critical aspect which must be considered during the design of the engine.

As far as the temperature fluctuations are concerned, the Entropy Waves (EWs) are timevarying total temperature variations due to an unsteady thermal release in the combustion process. These temperature fluctuations are convected through the combustor at the local

\footnotetext{
*Corresponding author: lorenzo.pinelli@tgroup.unifi.it
} 
speed of the flow and they are responsible for the generation of the indirect entropy noise during their acceleration within the turbine stage.

Nowadays, the study of entropy waves has become more and more important because of the effects on the thermal loading and the acoustic generation from the system [2]. In particular, the progressive reduction of jet noise has made entropy noise a relevant component of the overall acoustic emissions at the engine exhaust, thus this noise component has to be reduced in order to meet the future limitations in terms of noise emission levels [3, 4]. Moreover, the modern low- $\mathrm{NO}_{\mathrm{x}}$ combustor systems, operating with lean premixed flames are particular prone to the onset of combustion instabilities: when up-stream reflected pressure waves generated by convected entropy fluctuations interacts with the flame, they may lead to local time-varying temperature distortions and high-amplitude pressure fluctuations, with a risk of structural failures for the combustor liner and the turbine blades.

Since entropy waves are basically temperature fluctuations, their experimental characterization by means of a high-frequency temperature acquisition, could results very challenging because of the limited frequency response of the instrumentation [5]. On the other hand for numerical simulations, the study of entropy wave interaction with HPT stages needs for dedicated post processing approach based on the DFT of conservative variables in order to decompose the overall flow fluctuations and to extract the perturbations at the EWs frequencies $[6,7]$. As a matter of fact, the generated EWs can be considered sinusoidal fluctuations [5] and they usually produce a first harmonic response at the entropy wave frequency (EWF) during their evolution within the stage [7].

In order to study the clocking effect of the entropy wave spots respect to the stator leading edge, four simulations were performed, each one with a different circumferential position of the injections. More in detail LE (Leading Edge) case, MP (Mid Passage) case, SS (Suction Side) case and PS (Pressure Side) case were investigated, with the EWs injected on the LE of the stator blade, at mid pitch of the stator vane, at $1 / 3$ of pitch and $2 / 3$ of pitch respectively.

The excellent agreement between numerical results and experimental measurements represents a validation of the post-processing approach and it lays the foundations for furthers investigations about indirect noise generation due to entropy waves.

\section{Experimental Setup}

To prove the accuracy of the URANS simulations, the numerical results were compared with the experimental data acquired at the LFM of Politecnico di Milano.

The test rig is composed of a single HPT stage with 22 leaned stators $\left(10^{\circ}\right.$ on the pressure side) and 25 rotors rotating at $7000 \mathrm{rpm}$; for this experimental campaign the HPT stage operated at subsonic conditions and with $\mathrm{Tu}=2.5 \%$ at the inlet. Table 1 resumes the geometrical characteristics and the operating conditions of the stage.

Table 1. Geometry and operation conditions of the stage

\begin{tabular}{lccccccc}
\hline & $\begin{array}{c}\text { Blade } \\
\text { count }\end{array}$ & $\begin{array}{c}\text { Rotational } \\
\text { speed }\end{array}$ & $\begin{array}{c}\text { Exit Mach } \\
\text { number }\end{array}$ & $\begin{array}{c}\text { Exit Reynolds } \\
\text { number }\end{array}$ & $\boldsymbol{\beta}$ (stage) & Inlet $\boldsymbol{T}_{\boldsymbol{t}}$ & Tu \\
\hline Stator & 22 & - & 0.59 & $0.87 \times 10^{6}$ & 1.4 & $323[\mathrm{~K}]$ & $2.5 \%$ \\
Rotor & 25 & $7000[\mathrm{rpm}]$ & 0.45 & $0.50 \times 10^{6}$ & & \\
\hline
\end{tabular}

Figure 1 shows the sketch of the rig meridional cut (Figure 1a), the downstream view of the stage (Figure 1b) and the detail of airfoils stacking for stator and rotor blades (Figure 1c). The experimental data are acquired at planes T0, T1 and T2 reported in Figure 1a: the T0 plane is placed one stator axial-chord upstream the stage, the T1 plane is at a distance of $32 \%$ of stator axial chord downstream stator TE, and the T2 plane is at a distance of $32 \%$ rotor 
axial chord downstream the rotors. The description of the test rig and the measurements techniques are reported in $[5,8]$.

The Entropy Wave Generator (EWG) generates the EWs by the injection of jets at higher temperature through 11 injectors placed two axial chords upstream the stage; their azimuthal position can be varied to study the effect of different EW clocking positions. In order to reduce pressure disturbance connected to the flow injection, the hot fluid is injected in mechanical equilibrium with respect to the mainstream, resulting in a slightly different velocity for the two flows. Consequently, the shear stresses, added by the wake and the wall thickness effect of the injectors, increased turbulence level of about one order of magnitude in the injection region, promoting the diffusion of EWs.

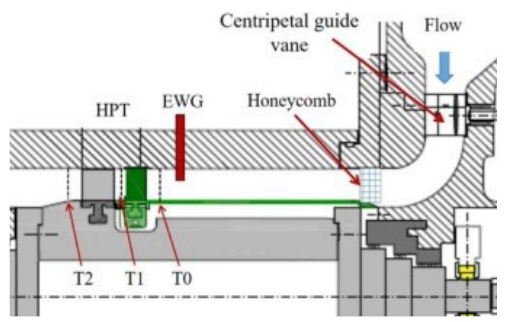

(a) Meridional view

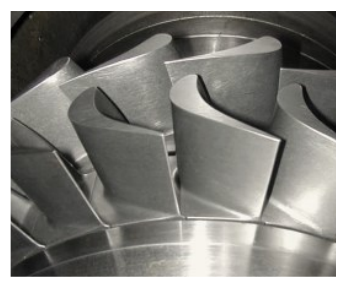

(b) Stage picture

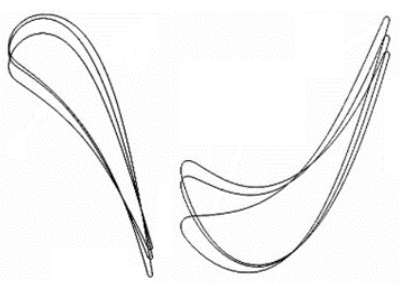

(c) Blade geometry

Figure 1. Rig description

Total temperature fluctuations at the inlet are nearly sinusoidal, as confirmed by experimental tests, and the generated EWs had a frequency of $90 \mathrm{~Hz}$, an amplitude of the $7 \%$ of the inlet total temperature $(\approx 19 \mathrm{~K})$ and a circular shape.

All these characteristics can be considered representative of the entropy waves which could arise in a real gas turbine combustor. Temperature measurements are performed by $\mu-$ thermocouple with a promptness of $200 \mathrm{~Hz}$, able to acquire the EW fluctuation at $90 \mathrm{~Hz}$.

\section{Numerical Setup}

For this study two different types of CFD URANS simulations were performed: a nodisturbance simulation (with uniform total temperature distribution at the inlet) and four simulations with entering EWs. The TRAF code, developed at the University of Florence [9], was used for all the analyses. This viscous code solves three dimensional Reynolds-averaged equation and it is based on finite volume formulation on multi-block structured $\mathrm{O}$-grids and H-grids. For the discretization of convective fluxes a $2^{\text {nd }}$ order Roe's upwind scheme based on TVD-MUSCL strategy was implemented; for diffusive fluxes a central difference scheme was adopted. The $k-\omega$ Wilcox's turbulence model in its high-Reynolds formulation was chosen for turbulence closure. For time discretization a dual time-stepping on 4-step RungeKutta scheme based on multigrid approach was used; 625 total physical time-steps for a single turbine revolution was selected in order to correctly solve the first and the second Blade Passing Frequency (BPF) harmonics.

Figure 2a shows the computational domain extension, the position of T0, T1 and T2 planes and the blocks where the DFT is computed to extract EW total temperature are highlighted. The inlet boundary corresponds to the T0 plane where total temperature fluctuations were measured; at the domain exit a buffer zone is added in order to prevent spurious reflections of acoustic waves on the outlet boundary [10]. The discretization of stator and rotor passages is based on elliptic structured O-grids as shown in Figure 2b, where the four different angular positions of the injections are also included. The stator mesh has $424 \times 64 \times 88$ cells along the blade, in pitch-wise direction and in span-wise direction 
respectively in order to ensure 30/40 cells for the shorter wavelength to be analysed. Following the same rule, the rotor mesh is defined by $448 \times 64 \times 88$ cells; the cell dimension in normal-wise direction on blade surface satisfies the condition of $y^{+}<2$ for boundary layer solution.

At the inlet and the exit of the stage, as well as between the two blade rows, H-grid blocks are added, for a total count of 8 blocks in axial direction. Since the numbers of stators and rotors are prime number each-other, all the five URANS simulations were run on the complete full-anulus domain, leading to a total cell number of about 200 millions.

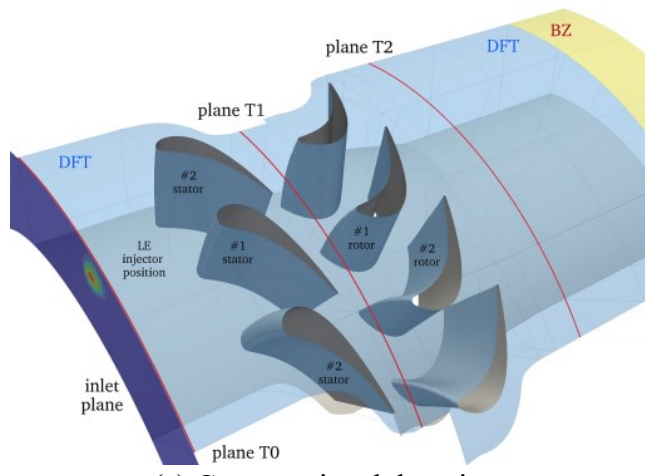

(a) Computational domain

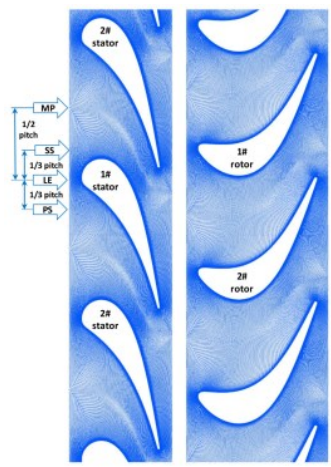

(b) Stator and rotor grids

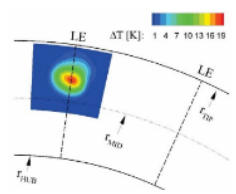

(c) Total temperature

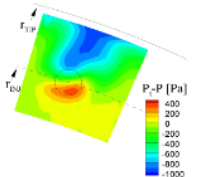

(d) Total pressure

Figure 2. Spatial discretization of the stage and incoming disturbance

The introduction of EWs in the domain was achieved with a $2 \mathrm{D}$ time-varying total temperature distributions at the inlet (Figure 2c) with 11 sinusoidal pulsating spots by imposing the instantaneous temperature levels. The entropy wave frequency was increase from $90 \mathrm{~Hz}$ (frequency of the experimental tests) to $116 \mathrm{~Hz}$ in order to have a single temperature fluctuation for each period of rotor revolution of the turbine at $7000 \mathrm{rpm}$ to reduce simulated time. To take in account the presence of injector wake, a 2D total pressure distribution was also imposed in inlet (Figure $2 \mathrm{~d}$ ). In the injection region a value of $\mathrm{Tu}=8 \%$ was imposed at the inlet following the experimental evidences. In order to reach a periodic solution, a total physical time corresponding to four revolution periods was simulated for each of five cases.

During the last period of simulation, a DFT algorithm directly implemented in the TRAF code was activated to extract the DFT coefficients of the conservative variables at the selected frequencies (EWF and BPFs) for the selected blocks.

\subsection{Unsteady post-processing}

Figure 3 shows the unsteady loads of a pair of stators and rotors blades for the LE case during a period of turbine revolution (\#1 is referred to the stator closer to the entropy wave spot, \#2 is related to the adjacent stator). As for \#1 stator, the entropy waves causes a modulation (shown by the dashed line) of the unsteady load characterized by the BPF, while the injector wake is responsible for the reduction in the mean value. The unsteady lift is nearly the same for the two rotor blades.

The load spectra (Figure 3b-c and Figure 3e-f) show that the variation of pressure field on blade surfaces is due to the first and second harmonics at $1 \times \mathrm{BPF}$ and $2 \times \mathrm{BPF}$ defined by their Engine Order (EO) values (corresponding at $\mathrm{EO}=25$ and $\mathrm{EO}=50$ for the stators and at $\mathrm{EO}=22$ and $\mathrm{EO}=44$ for the rotors) and to the first $\mathrm{EW}$ harmonic $(\mathrm{EO}=1)$. The effect of the second harmonic of the entropy waves $(\mathrm{EO}=2)$ is negligible confirming the sinusoidal trend. 
The analysis of the unsteady load spectra leads to the conclusion that the fluctuations of flow quantities due to the presence of EWs can be accurately described by the first harmonic.

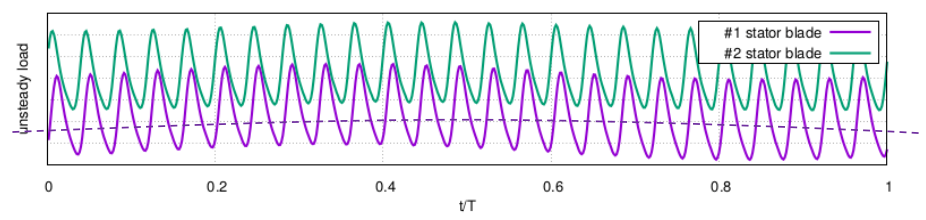

(a) Stator unsteady lift

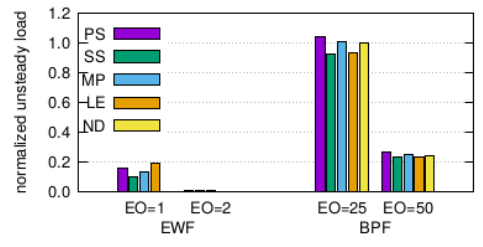

(b) Stator lift spectrum (1\# stator)

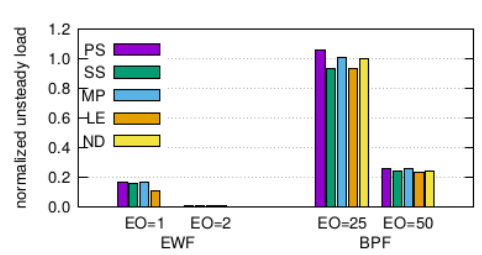

(c) Stator lift spectrum (2\# stator)

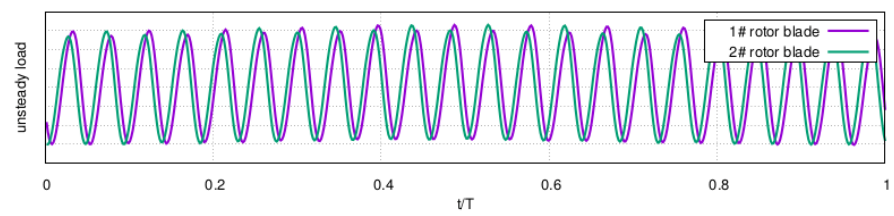

(d) Rotor unsteady lift

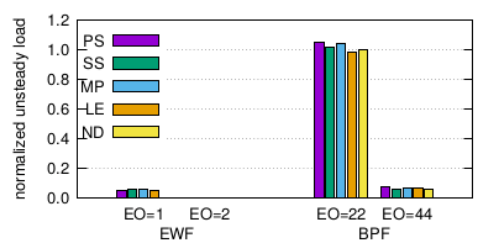

(e) Rotor lift spectrum (1\# rotor)

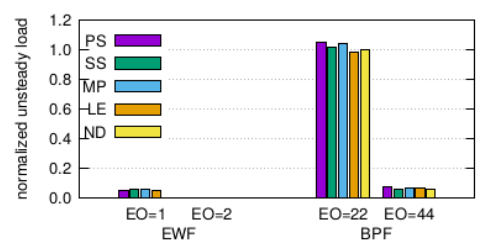

(f) Rotor lift spectrum (2\# rotor)

Figure 3. Stator and rotor loads

Therefore, they can be expressed by phasors coming from the Discrete Fourier Transform of conservative variables at the $\mathrm{EWF}(\mathrm{EO}=1)$ :

$$
\underline{\overline{\bar{U}}}^{(1)}=\underline{U}_{\delta}=\left(\rho_{\delta},\left(\rho c_{x}\right)_{\delta},\left(\rho c_{y}\right)_{\delta^{\prime}}\left(\rho c_{z}\right)_{\delta},\left(\rho c_{t}\right)_{\delta}\right)
$$

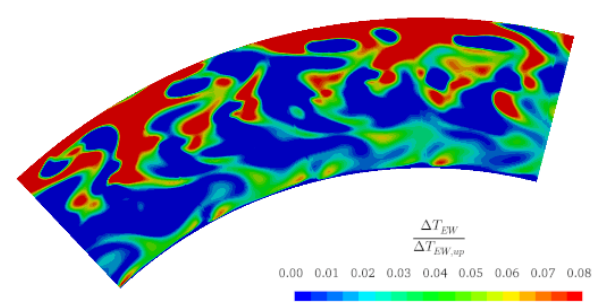

(a) Instantaneous fluctuation

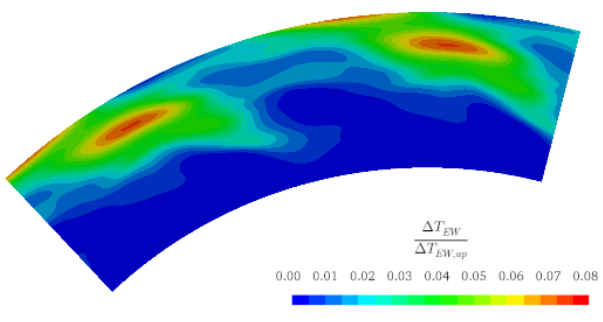

(b) Filtered fluctuation

Figure 4. Total temperature fluctuation at the rotor exit (MP case, $\mathrm{t} / \mathrm{T}=0.50$ )

From a numerical point of view, the total temperature fluctuations due to the EWs can be computed as a rotating phasor (that is the Fourier coefficient) at the EWF $(116 \mathrm{~Hz})$ by the formula: 


$$
T_{t \delta}=T_{s \delta}+\frac{\gamma-1}{R \gamma}\left(\bar{c}_{x} c_{x \delta}+\bar{c}_{y} c_{\mathrm{y} \delta}+\bar{c}_{z} c_{z \delta}\right)
$$

The formula derivation is described in [7].

Figure 4 makes clear the need for this dedicated post-processing approach to follow the evolution of EWs. The phasor approach enables to isolate the total temperature disturbance at $116 \mathrm{~Hz}$ introduced by the EWs and to reproduce the actual experimental acquisition by filtering temperature disturbance at higher frequency (for example those due to stator-rotor interaction) which are not detected by the thermocouples having a promptness of $200 \mathrm{~Hz}$.

\section{Results}

The results of the simulations and the comparisons with experimental measurements are presented in this section, focusing on instantaneous temperature field distortions and secondary flow modification due to EWs with respect to the no-disturbance case.

\subsection{No-Disturbance Simulation}

Before showing the EW evolution, it is important to recall the main aerodynamic characteristics of the stage, already discussed in detail in a previous work by the authors [5][8].

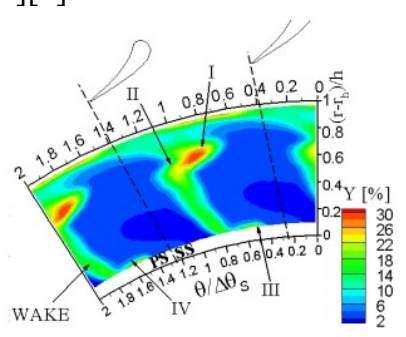

(a) Total pressure loss coeff. (EXP)

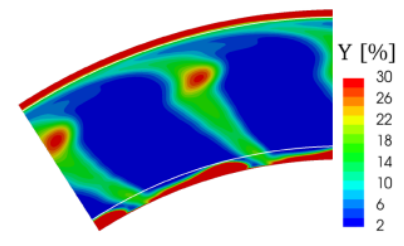

(b) Total pressure loss coeff. (TRAF)

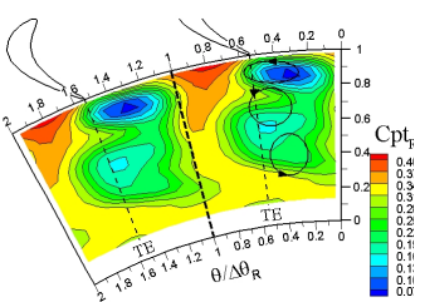

(c) Relative pressure loss coeff. (EXP)

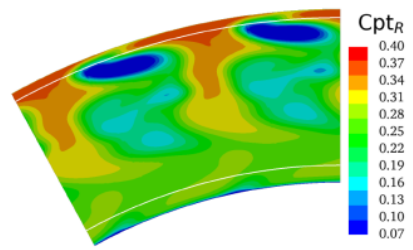

(d) Relative pressure loss coeff. (TRAF)

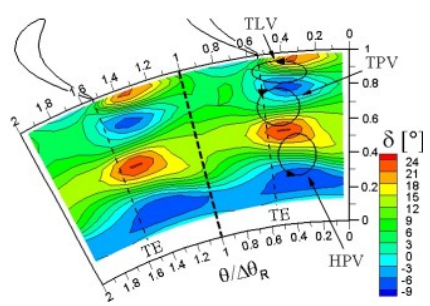

(e) Relative deviation angle (EXP)

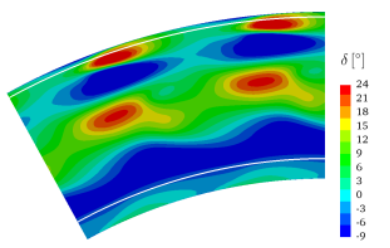

(f) Relative deviation angle (TRAF)

Figure 5. No-disturbance results

Figure 5 shows the comparisons of the no-disturbance simulations with the experiments in terms of the total pressure loss coefficient downstream the stator (Figure 5a-b), the relative total pressure coefficient downstream the rotor (Figure $5 \mathrm{c}-\mathrm{d}$ ) and the deviation angle at the rotor exit (Figure 5e-f). These quantities are related to the vortex structures and secondary flows in both stator and rotor vanes, which are responsible of entropy wave migration towards the hub region of the rotor [11]. The good agreement between numerical results and the correspondent experimental data proves the accuracy of the numerical simulation and the capability of TRAF code to describe the development of secondary flows and therefore the principal migration mechanism of the EWs within the rotor. 


\subsection{Entropy wave simulations}

\subsubsection{Stator exit - plane $T_{1}$}

Figure 6 reports the amplitude of the total temperature variation at the stator exit for the four injection positions: the contours show the temperature increase due to entropy waves with respect to no-disturbance case $\left(\Delta T_{E W}\right)$ normalized with the inlet maximum total temperature distortion $\Delta T_{E W, u p}=19 \mathrm{~K}$.

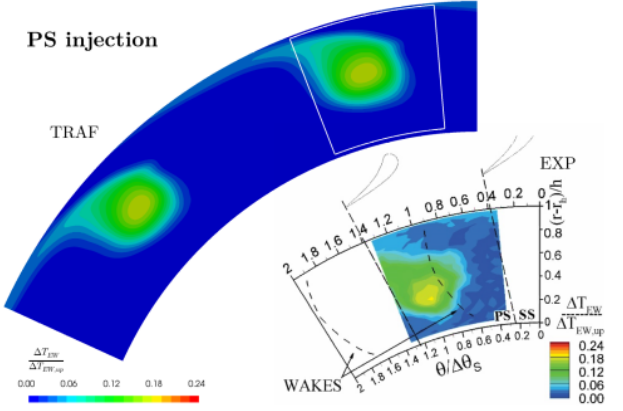

(a) PS case

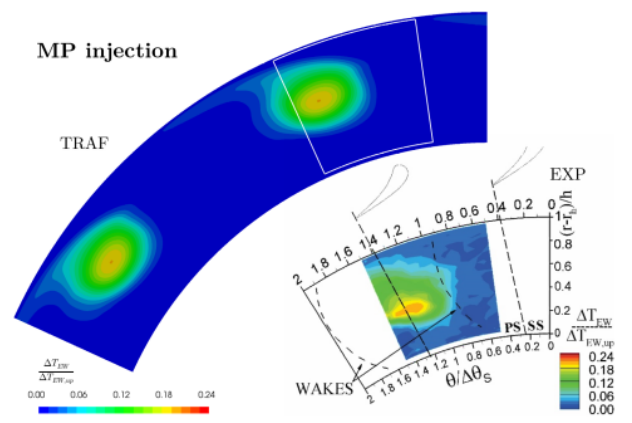

(c) MP case

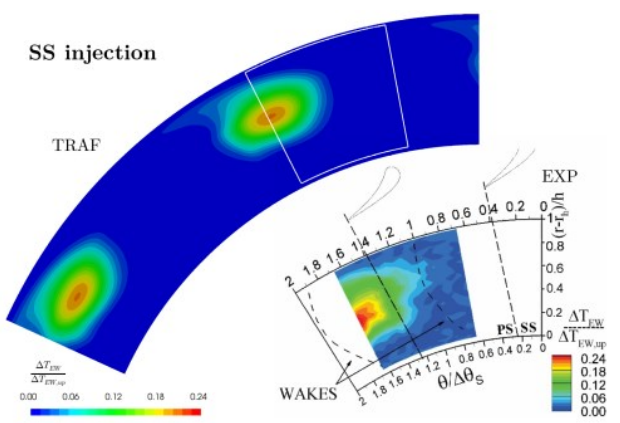

(b) SS case

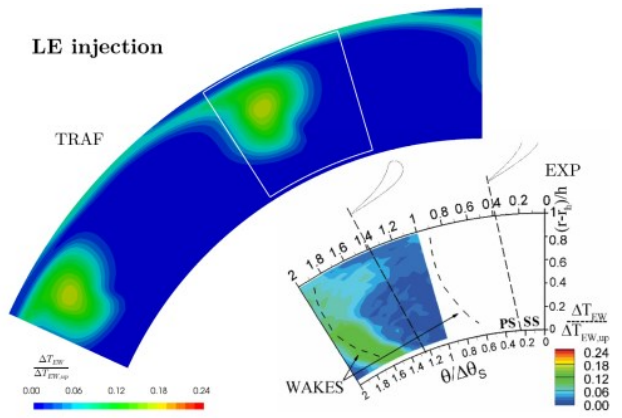

(a) LE case

Figure 6. Amplitude of the total temperature distortions at the stator exit

The numerical results are in good agreement with experimental measurements: for all cases the simulations are able to describe the temperature attenuation of the disturbance, the shape of the spot and its radial migration tendency. Nevertheless, the CFD simulations tend to underestimate the spot diffusion, especially for the LE injection case.

The radial migration of the spot is related to the EW clocking and it can be seen that the transport of entropy waves within the stator vanes is heavily affected by the spot interaction with the stator pressure field. Because of the opposite radial pressure gradient on PS and SS due to the stator lean angle, the spot is pushed towards the hub region when entropy waves are injected near the pressure side, on the contrary it migrates towards the tip in the SS case. In the MP case, the spot shows an intermediate behaviour between SS and PS injection. LE case is the one that differs the most from experimental measurements because of the most complex interaction between the EWs and the stator blades: for this position, the spot is cut by the blade leading edge.

As for temperature fluctuation drop, the predicted attenuation of about $3-4 \mathrm{~K}$ at the stator exit is in agreement with experimental measurements. 


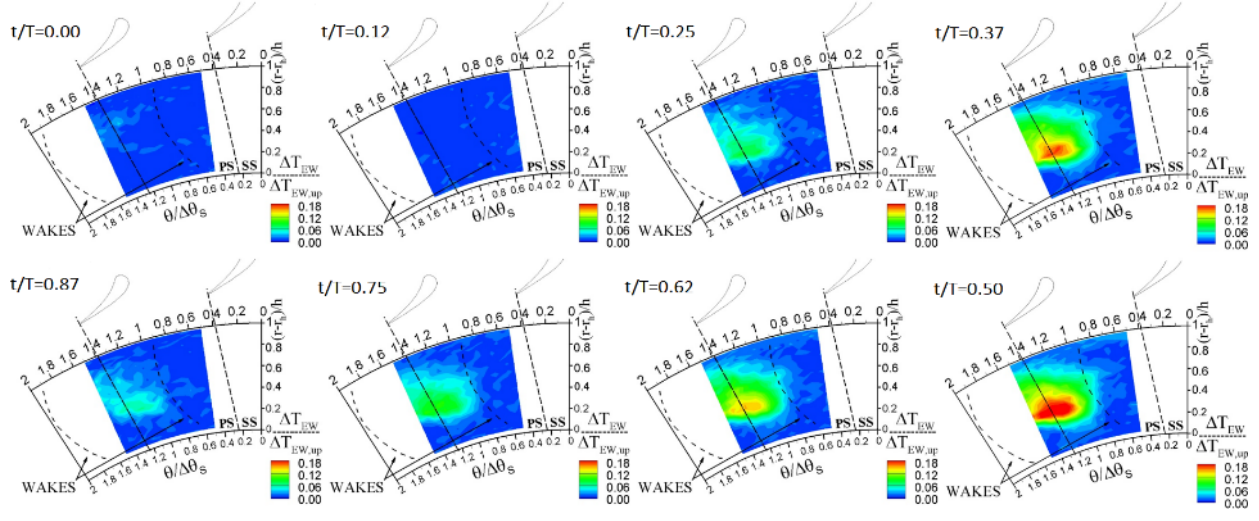

(a) EXP

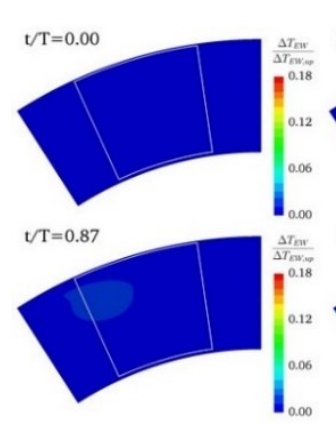

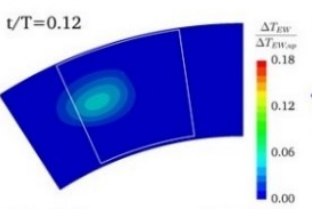
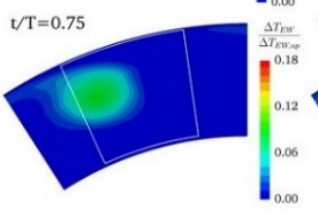

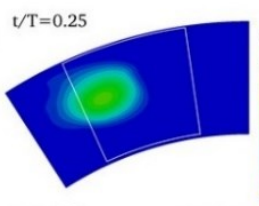

$\mathrm{t} / \mathrm{T}=0.62$

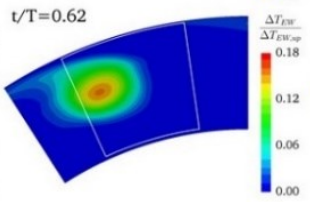

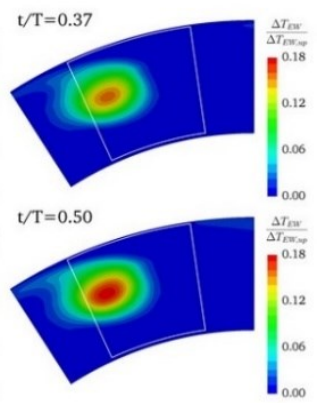

(b) TRAF

Figure 7. Time evolution of EW at stator exit for MP case: experimental contours (a) and numerical results (b)

As an example, Figure 7 shows the entropy wave time evolution at T1 plane for MP case in terms of normalized temperature increase due to entropy spots in 8 equally spaced instants within a fluctuation period. The instantaneous values of total temperature fluctuation are obtained from the real part of the rotating phasor $T_{t \delta}\left(T_{t}(t)=\operatorname{Re}\left[T_{t \delta} e^{j \omega t}\right]\right.$, where $\omega=$ $2 \pi \cdot \mathrm{EWF})$.

The results of the numerical simulations (Figure $7 \mathrm{~b}$ ) are generally in good agreement with the corresponding experimental measurements (Figure 7a) in terms of spot shape and temperature values for all the instants, especially for the instant $t / T=0.5$ where the temperature disturbance reaches its maximum.

\subsubsection{Rotor exit - plane $T_{2}$}

The amplitude of the total temperature distortion at the rotor exit is shown in Figure 8 for the four different injection positions. The range of depicted contours is about the half of that use in $\mathrm{T} 1$ plane because of the further attenuation through the rotor.

The excellent agreement between numerical results and experimental measurements for all cases proves the accuracy of the CFD simulations and validates the DFT-based postprocessing approach for the study of EW evolution. 


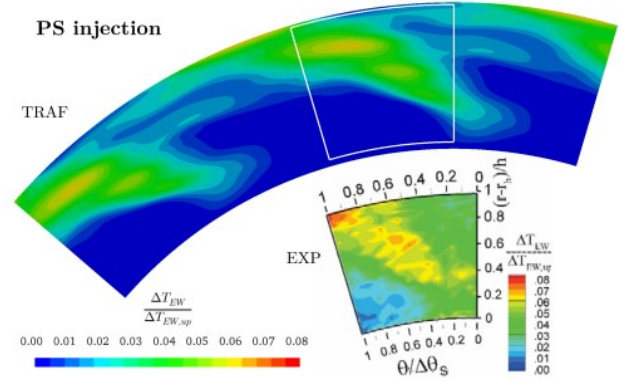

(a) PS case

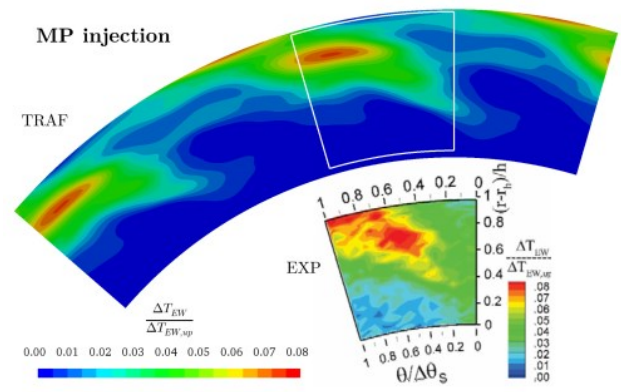

(c) MP case

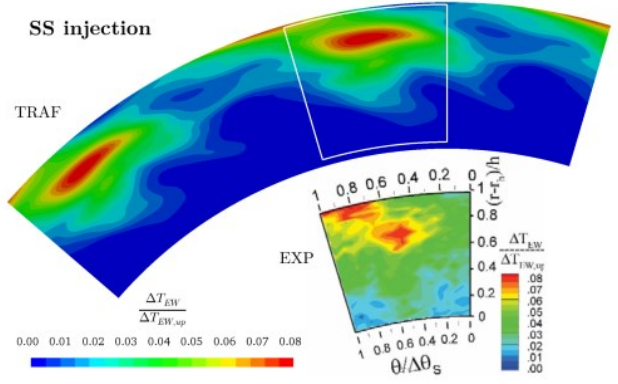

(b) SS case

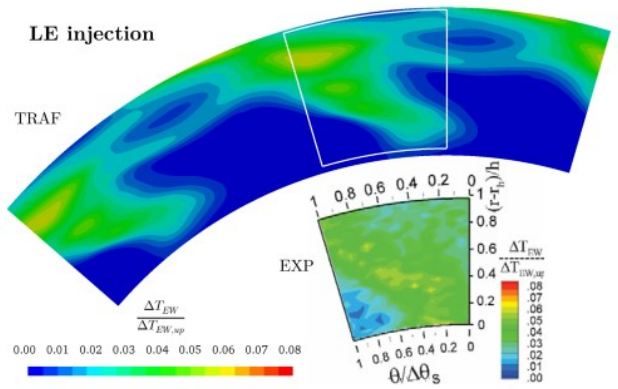

(a) LE case

Figure 8. Amplitude of the total temperature distortions at the rotor exit

Moreover, the very good matching with the experimental contours suggests that the interaction of the spots with the rotor is the main mechanism that affects EW evolution through the stage, while the contribution of diffusion, which is underestimated by CFD calculations, is globally less important. Generally speaking, the spot shape at rotor exit is not nearly circular as it was at the stator exit, but it spreads in circumferential and in spanwise directions. The SS and MP case shows the higher temperature level, in agreement with the results found in $\mathrm{T} 1$ plane.

Figure 9 shows EW time evolution at the rotor exit for MP case in 4 equally spaced instants within a period of fluctuations. The numerical results (Figure 9b) are obtained by the real part of the phasor $T_{t \delta}$ and the agreement with the instantaneous measured temperature levels is very good for all the frames. The evolution of the spot shape and the temperature disturbance values are well predicted by the simulation for all the instants. It is worth considering that this DFT-based post-processing succeeded to extract the measured temperature fluctuations at the rotor exit with an amplitude $\left|T_{t \delta}\right|<2 \mathrm{~K}$ which would not be visible otherwise by making a point-by-point difference between the instantaneous temperature fields with and without EWs.

An interesting aspect to be studied is the interaction between EWs and the rotor secondary flows. In fact, the interaction with vortex structures, which are commonly present within the rotor, is the main mechanism of EW radial migration towards the hub region. Moreover, even if the temperature disturbance at inlet is low and its amplitude strongly decreases in its evolution within the stage, it is known by the literature [12] that a non-uniform total temperature distribution at the rotor inlet is responsible of total relative pressure gradients, and therefore of non-conventional secondary flows development within rotor vane. 

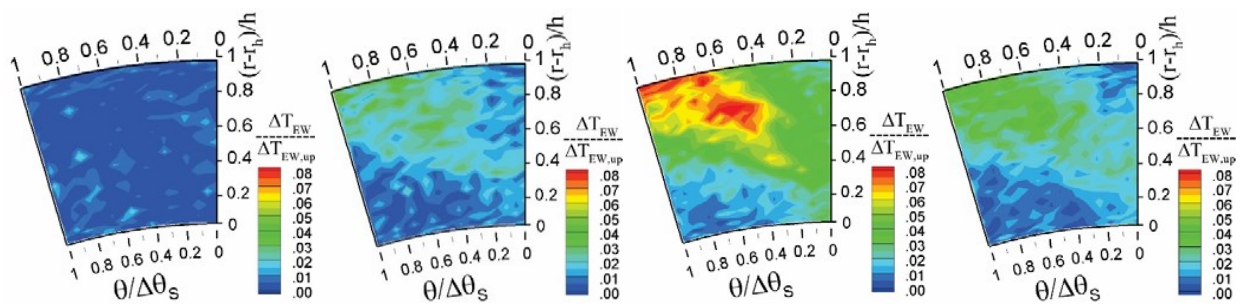

(a) EXP
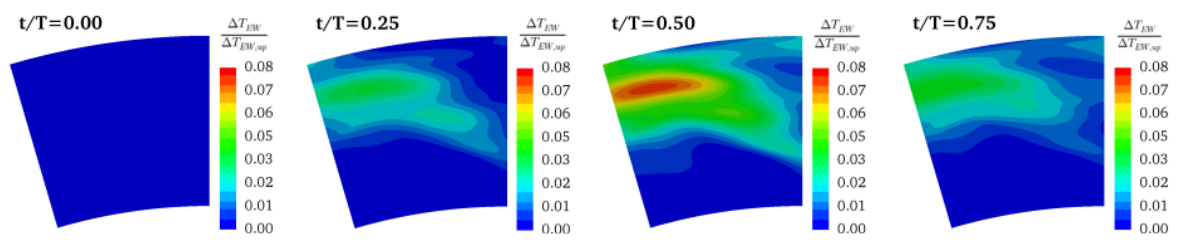

(b) TRAF

Figure 9. Time evolution of $\mathrm{EW}$ at stator exit for MP case

To aid readers' understanding, Figure 10 show the deviation angle at rotor exit for nodisturbance and SS case. The EW passage causes a reduction of deviation angle at midspan and this effect is confirmed by the q-criterion iso-contours coloured with axial vorticity and EW path in terms of $\left|T_{t \delta}\right|$ in orange for no-disturbance and SS cases (see Figure 11). The passage of the spot at higher temperature reduces the intensity of secondary flows at midspan; on the contrary vortex structures are not altered in the hub region.

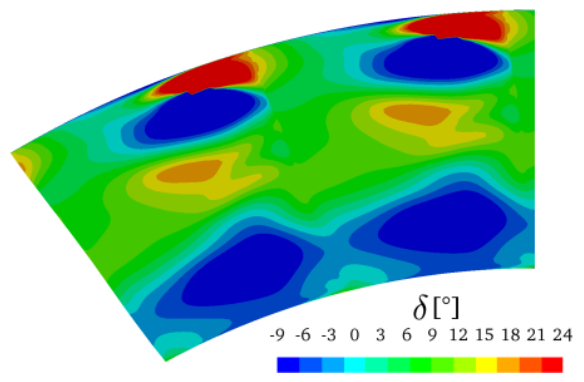

(a) no-disturbance

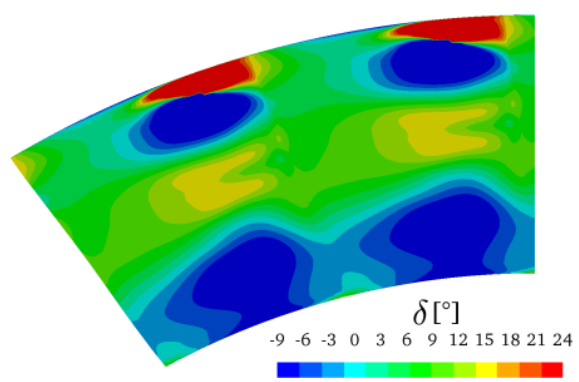

(b) SS case

Figure 10. Deviation angle $\delta$ at the rotor exit

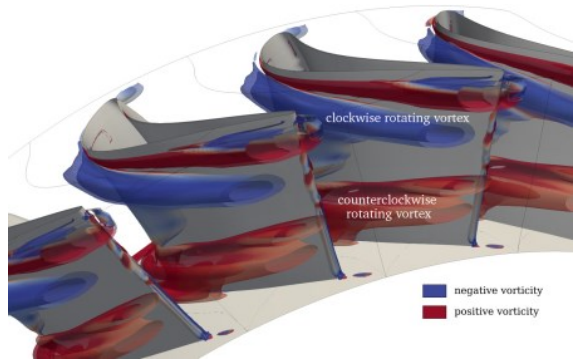

(a) no-disturbance

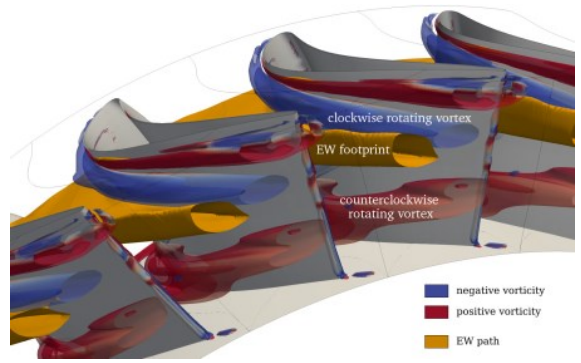

(b) SS case

Figure 11. Secondary flows in the rotor passage 


\section{CONCLUSIONS}

Combustor-turbine interaction is a crucial aspect in aero-engine design because of the mutual effects produced by the combustion process flow distortions with turbine stages. Moreover, this interaction produces indirect entropy noise which must be reduced in order to meet future goals in terms of noise emission limits.

To study the evolution of EWs within a HP turbine stage two different types of CFD URANS simulations with and without total temperature disturbance were performed and the numerical results were compared with experimental measurements taken at the Lab. of Fluid Machines (LFM) of the Politecnico di Milano. For the EW simulations a 2D time-varying total temperature distribution was imposed at the inlet for different clocking position of the 11 injectors, respectively the LE, MP, PS and SS cases. To follow the EW evolution within the stage a dedicated post-processing, based on DFT, was implemented in order to decompose the overall fluctuations and to isolate the EW contribution at low frequency. The total temperature fluctuation related to the EW is described by the phasor quantity $T_{t \delta}$ and the results are presented in terms of total temperature fluctuations, normalized with respect to the no-disturbance case.

At the stator exit, numerical results are in good agreement with experiments for all the injection positions: the simulations were able to describe the spot tendency to migrate in radial direction and the maximum temperature attenuation of about $4 \mathrm{~K}$. Nevertheless, the spot diffusion is underestimated by numerical simulations, in particular for the LE case.

The numerical results at rotor exit are in excellent agreement with experimental measurements: both spot shape and temperature levels are captured by simulations. The very good correspondence with experimental data confirmed the accuracy of CFD simulations. Moreover, it can be seen that the spot interaction with rotor blades is the main mechanism of the EW evolution within the stage. Since a total temperature inhomogeneity at the rotor inlet can cause non-conventional secondary flows, the vortex structures within the rotor vanes was investigated to highlight the effect of the temperature spots. Both the deviation angle contours at the rotor exit and the iso-contours of q-criterion showed that the entropy wave causes a reduction of secondary flows intensity around mid-span.

In conclusion, the combination of experimental measurements and numerical results provided interesting information about EWs evolution respect to different clocking positions which can be essential in the design of the HPT stages. This study is also preparatory for the investigation of indirect entropy noise generation, caused by the EW acceleration in the turbine stage.

\section{REFERENCES}

1. Lefebvre A. H. and Ballal D. R., 2010, "Gas turbine combustion: in Alternative fuels and emissions", in Boca Raton: Taylor \& Francis.

2. Knoblock K., Neuhaus L., Bake F., Gaetani P. and Persico G., 2017, "Experimental Assessment of Noise Generation and Transmission in a High-Pressure Transonic Turbine Stage", in ASME Journal of Turbomachinery, 139, 101006.

3. Morgans A. S. and Durán I., 2016, "Entropy Noise: A Review of Theory, Progress and Challenges", in International Journal of Spray and Combustion Dynamics, 8, pp. 285 298.

4. Pinelli L. et al., 2015, "On the Numerical Evaluation of Tone Noise Emissions Generated by a Turbine Stage: An In-Depth Comparison Among Different Computational Methods", in ASME Turbo Expo: Power for Land, Sea, and Air, Vol. 2B: Turbomachinery, pp. V02BT41A002, doi:10.1115/GT2015-42376. 
5. Gaetani, P. and Persico, G., 2018, "Transport of Entropy Waves Within a High Pressure Turbine Stage", in ASME Journal of Turbomachinery, 141(3): 031006, https://doi.org/10.1115/1.4042165.

6. Bicchi M., Pinelli L., Marconcini M., Gaetani P., and Persico G., 2019, "Numerical Study of a High-Pressure Turbine Stage with Inlet Distortions", in AIP Conference Proceedings, 2191, 10.1063/1.5138753.

7. Pinelli L., Marconcini M., Pacciani R., Gaetani P. and Persico G., 2020, "Computational and Experimental Study of the Unsteady Convection of Entropy Waves Within a High Pressure Turbine Stage", in ASME Turbo Expo 2020 Turbomachinery Technical Conference and Exposition.

8. Gaetani P., Persico G., Pinelli L., Marconcini M. and Pacciani R., 2020 "Computational and Experimental Study of Hot Streak Transport within the First Stage of a Gas Turbine," in ASME Journal of Turbomachinery, 142(8): 081002. https://doi.org/10.1115/1.4045961

9. Arnone A., 1994, "Viscous analysis of three-dimensional rotor flow using a multigrid method", in ASME Journal of Turbomachinery, 116, pp. 435-445.

10. Burberi C., Ghignoni E., Pinelli L. and Marconcini M., 2018, "Numerical Analysis of Direct and Indirect Noise Produced by a High Pressure Turbine Stage" in ATI 2018 73rd Conference of the Italian Thermal Machines Engineering Association, Energy Procedia, 148:130-137.

11. Ong J. and Miller R. J., 2012, "Hot Streak and Vane Coolant Migration in a Downstream Rotor", in ASME Journal of Turbomachinery, Vol. 134 (5).

12. Hawthorne W. R., 1974, "Secondary vorticity in stratified compressible fluids in rotating systems," in CUED/A-Turbo/TR 63, University of Cambridge, Cambridge, UK. 